\title{
PARTIAL SUMS OF CERTAIN ANALYTIC FUNCTIONS
}

\section{SHIGEYOSHI OWA}

(Received 3 May 2000)

AвSTRACT. The object of the present paper is to consider the starlikeness and convexity of partial sums of certain analytic functions in the open unit disk.

2000 Mathematics Subject Classification. Primary 30C45.

1. Introduction. Let $A$ denote the class of functions $f(z)$ of the form

$$
f(z)=z+\sum_{k=2}^{\infty} a_{k} z^{k},
$$

which are analytic in the open unit disk $U=\{z \in \mathbb{C}:|z|<1\}$. Let $S^{*}(\alpha)$ be the subclass of $A$ consisting of functions $f(z)$ which satisfy

$$
\operatorname{Re}\left[\frac{z f^{\prime}(z)}{f(z)}\right]>\alpha \quad(z \in U)
$$

for some $\alpha(0 \leq \alpha<1)$. A function $f(z)$ in $S^{*}(\alpha)$ is said to be starlike of order $\alpha$ in $U$. Furthermore, let $K(\alpha)$ denote the subclass of $A$ consisting of all functions $f(z)$ which satisfy

$$
\operatorname{Re}\left[1+\frac{z f^{\prime \prime}(z)}{f^{\prime}(z)}\right]>\alpha \quad(z \in U)
$$

for some $\alpha(0 \leq \alpha<1)$. A function $f(z)$ belonging to $K(\alpha)$ is said to be convex of order $\alpha$ in $U$. We note that $f(z) \in S^{*}(\alpha)$ if and only if $z f^{\prime}(z) \in K(\alpha)$ and denote by $S^{*}(0) \equiv S^{*}$ and $K(0) \equiv K$. For $f(z) \in A$, we introduce the partial sum of $f(z)$ by

$$
f_{n}(z)=z+\sum_{k=2}^{n} a_{k} z^{k} .
$$

REMARK 1.1. It is well known that

(i) $f(z)=z /(1-z)^{2}=z+\sum_{k=2}^{\infty} k z^{k}$ is the extremal function for the class $S^{*}$. But $f_{2}(z)=z+2 z^{2} \notin S^{*}$.

(ii) $f(z)=z /(1-z)=z+\sum_{k=2}^{\infty} z^{k}$ is the extremal function for the class $K$. But $f_{2}(z)=z+z^{2} \notin K$.

For the partial sums $f_{n}(z)$ of $f(z) \in S^{*}$, Szegö [2] showed the following theorem. 
THEOREM 1.2. (i) $f(z) \in S^{*}$ implies that $f_{n}(z) \in S^{*}$ for $|z|<1 / 4$. The result is sharp. (ii) $f(z) \in S^{*}$ implies that $f_{n}(z) \in K$ for $|z|<1 / 8$. The result is sharp.

Further, Padmanabhan [1] proved the following theorem.

THEOREM 1.3. If $f(z)$ is 2-valently starlike in $U$, then $f_{n}(z)$ is 2-valently starlike for $|z|<1 / 6$. The result is sharp.

2. Function $F_{n}(z)$. We define the function $F_{n}(z)$ which is the partial sum of $f(z) \in$ $A$ by

$$
F_{n}(z)=z+a_{n} z^{n}
$$

THEOREM 2.1. The function $F_{n}(z)$ satisfies

$$
\frac{1-n\left|a_{n}\right| r^{n-1}}{1-\left|a_{n}\right| r^{n-1}} \leq \operatorname{Re}\left[\frac{z F_{n}^{\prime}(z)}{F_{n}(z)}\right] \leq \frac{1+n\left|a_{n}\right| r^{n-1}}{1+\left|a_{n}\right| r^{n-1}}
$$

for $0 \leq r<\sqrt[n-1]{1 /\left|a_{n}\right|} \leq 1$. Therefore, $F_{n}(z) \in S^{*}(\alpha)$ for $0 \leq r<\sqrt[n-1]{(1-\alpha) /(n-\alpha)\left|a_{n}\right|} \leq 1$.

Proof. Note that

$$
\frac{z F_{n}^{\prime}(z)}{F_{n}(z)}=\frac{z+n a_{n} z^{n}}{z+a_{n} z^{n}}=n-\frac{n-1}{1+a_{n} z^{n-1}} .
$$

It follows from (2.3) that

$$
\operatorname{Re}\left[\frac{z F_{n}^{\prime}(z)}{F_{n}(z)}\right]=n-(n-1) \frac{1+\left|a_{n}\right| r^{n-1} \cos \theta}{1+\left|a_{n}\right|^{2} r^{2(n-1)}+2\left|a_{n}\right| r^{n-1} \cos \theta} .
$$

Since, the right-hand side of (2.4) is increasing for $\cos \theta$ if $\left|a_{n}\right|<1$, we obtain (2.2). Further, we also see that

$$
\operatorname{Re}\left[\frac{z F_{n}^{\prime}(z)}{F_{n}(z)}\right] \geq \frac{1-n\left|a_{n}\right| r^{n-1}}{1-\left|a_{n}\right| r^{n-1}}>\alpha
$$

for $0 \leq r<\sqrt[n-1]{(1-\alpha) /(n-\alpha)\left|a_{n}\right|} \leq 1$. This completes the proof of the theorem.

Next, we derive the following theorem.

THEOREM 2.2. The function $F_{n}(z)$ satisfies

$$
\frac{1-n^{2}\left|a_{n}\right| r^{n-1}}{1-n\left|a_{n}\right| r^{n-1}} \leq \operatorname{Re}\left[1+\frac{z F_{n}^{\prime \prime}(z)}{F_{n}^{\prime}(z)}\right] \leq \frac{1+n^{2}\left|a_{n}\right| r^{n-1}}{1+n\left|a_{n}\right| r^{n-1}}
$$

for $0 \leq r<\sqrt[n-1]{1 / n\left|a_{n}\right|} \leq 1$. Therefore, $F_{n}(z) \in K$ for $0 \leq r<\sqrt[n-1]{(1-\alpha) / n(n-\alpha)\left|a_{n}\right|} \leq 1$.

Proof. Noting that

$$
1+\frac{z F_{n}^{\prime \prime}(z)}{F_{n}^{\prime}(z)}=n-\frac{n-1}{1+n a_{n} z^{n-1}},
$$

we have

$$
\operatorname{Re}\left[1+\frac{z F_{n}^{\prime \prime}(z)}{F_{n}^{\prime}(z)}\right]=n-(n-1) \frac{1+n\left|a_{n}\right| r^{n-1} \cos \theta}{1+n^{2}\left|a_{n}\right|^{2} r^{2(n-1)}+2 n\left|a_{n}\right| r^{n-1} \cos \theta},
$$

which derives (2.6). 
By virtue of Theorems 2.1 and 2.2, we have the following conjecture.

CONJECTURE 2.3. For the partial sum $f_{n}(z)$ of $f(z)$ belonging to the class $A$,

(i) $f_{n}(z) \in S^{*}(\alpha)$ for $0 \leq r<\sqrt[n-1]{(1-\alpha) /(n-\alpha)\left|a_{n}\right|} \leq 1$,

(ii) $f_{n}(z) \in K(\alpha)$ for $0 \leq r<\sqrt[n-1]{(1-\alpha) / n(n-\alpha) \mid a_{n}} \leq 1$.

3. The partial sums of certain analytic functions. In this section, we consider the partial sums of functions $f(z)=z /(1-z)$ and $f(z)=z /(1-z)^{2}$.

THEOREM 3.1. Let $f_{3}(z)=z+z^{2}+z^{3}$ be the partial sum of $f(z)=z /(1-z)$ which is the extremal function of the class $K$. Then $f_{3}(z) \in S^{*}(626 / 961)$ for $0 \leq r<\beta(1 / 7<$ $\beta<1 / 6)$, where $\beta$ is the positive root of

$$
x^{4}-8 x^{3}+9 x^{2}-8 x+1=0 \quad\left(0<x<\frac{1}{\sqrt{3}}\right) .
$$

Proof. We consider $\alpha$ such that

$$
\operatorname{Re}\left[\frac{z f_{3}^{\prime}(z)}{f_{3}(z)}\right]=\operatorname{Re}\left[3-\frac{2+z}{1+z^{2}+z^{3}}\right]>\alpha
$$

for $0 \leq r<\beta$. This implies that

$$
\operatorname{Re}\left[\frac{2+z}{1+z^{2}+z^{3}}\right]=1+\frac{\left(1-r^{2}\right)\left(1+r^{2}+r \cos \theta\right)}{1-r^{2}+r^{4}+4 r^{2} \cos ^{2} \theta+2 r\left(1+r^{2}\right) \cos \theta}<3-\alpha,
$$

that is,

$$
\operatorname{Re}\left[\frac{\left(1-r^{2}\right)\left(1+r^{2}+r \cos \theta\right)}{1-r^{2}+r^{4}+4 r^{2} \cos ^{2} \theta+2 r\left(1+r^{2}\right) \cos \theta}\right]<2-\alpha .
$$

Let the function $g(t)$ be given by

$$
g(t)=\frac{\left(1-r^{2}\right)\left(1+r^{2}+r t\right)}{1-r^{2}+r^{4}+4 r^{2} t^{2}+2 r\left(1+r^{2}\right) t} \quad(t=\cos \theta) .
$$

Then, we have

$$
g^{\prime}(t)=\frac{r(r+1)(r-1)\left(1+5 r^{2}+r^{4}+4 r^{2} t^{2}+8 r\left(1+r^{2}\right) t\right)}{\left(1-r^{2}+r^{4}+4 r^{2} t^{2}+2 r\left(1+r^{2}\right) t\right)^{2}} .
$$

Letting

$$
h(t)=1+5 r^{2}+r^{4}+4 r^{2} t^{2}+8 r\left(1+r^{2}\right) t,
$$

we see that (i) $h(t)<0 \Rightarrow g^{\prime}(t)>0$, (ii) $h(t)>0 \Rightarrow g^{\prime}(t)<0$, and (iii) $h(t)=0$ for $t=\left(-2\left(1+r^{2}\right) \pm \sqrt{3\left(1+r^{2}+r^{4}\right)}\right) / 2 r$.

If we write

$$
t_{1}=\frac{-2\left(1+r^{2}\right)+\sqrt{3\left(1+r^{2}+r^{4}\right)}}{2 r}<0,
$$

then, $0 \leq r \leq \beta$ implies that $t_{1} \leq-1$, so that, $h(t) \geq 0$. This gives us that

$$
g(t) \leq g(-1)=\frac{1-r+r^{3}-r^{4}}{1-2 r+3 r^{2}-2 r^{3}+r^{4}}=\frac{g_{1}(r)}{g_{2}(r)} .
$$


It is easy to check that $g_{1}(r)$ is decreasing for $r(0 \leq r<1 / \sqrt{3})$. Therefore,

$$
\frac{8-2 \sqrt{3}}{9}=g_{1}\left(\frac{1}{\sqrt{3}}\right)<g_{1}(r) \leq g_{1}(0)=1 .
$$

Also, $g_{2}(r)$ is decreasing for $r(0 \leq r<\beta)$, because $g_{2}^{\prime}(0)=-2<0$ and $g_{2}^{\prime}(1 / 6)=$ $-31 / 27<0$. This gives that

$$
\frac{961}{1296}=g_{2}\left(\frac{1}{6}\right)<g_{2}(r) \leq 1 .
$$

Consequently, we conclude that

$$
g(t) \leq g(-1)=\frac{g_{1}(r)}{g_{2}(r)}<\frac{1296}{961}=2-\alpha,
$$

that is, $\alpha=626 / 961=0.651 \ldots$ Thus, we have

$$
\operatorname{Re}\left[\frac{z f_{3}^{\prime}(z)}{f_{3}(z)}\right]>\alpha \quad\left(\alpha=\frac{626}{961}\right)
$$

for $0 \leq r<\beta$.

Finally, we obtain the following theorem.

THEOREM 3.2. Let $f_{3}(z)=z+2 z^{2}+3 z^{3}$ be the partial sum of the Koebe function $f(z)=z /(1-z)^{2}$ which is the extremal function for the class $S^{*}$. Then $f_{3}(z) \in$ $K(3191 / 15876)$ for $0 \leq r<\beta(1 / 14<\beta<113)$, where $\beta$ is the positive root of

$$
81 x^{4}-162 x^{3}+72 x^{2}-18 x+1=0 \quad\left(0 \leq x<\frac{1}{3}\right) .
$$

Proof. Since

$$
\operatorname{Re}\left[1+\frac{z f_{3}^{\prime \prime}(z)}{f_{3}^{\prime}(z)}\right]=\operatorname{Re}\left[3-\frac{2(1+2 z)}{1+4 z+9 z^{2}}\right]>\alpha
$$

implies that

$$
\begin{aligned}
\operatorname{Re}\left[\frac{1+2 z}{1+4 z+9 z^{2}}\right] & =\frac{1}{2}+\frac{4 r\left(1-9 r^{2}\right) \cos \theta+1-81 r^{4}}{2\left(1-2 r^{2}+81 r^{4}+8 r\left(1+9 r^{2}\right) \cos \theta+36 r^{2} \cos ^{2} \theta\right)} \\
& <\frac{3-\alpha}{2},
\end{aligned}
$$

we have to check that

$$
\frac{\left(1-9 r^{2}\right)\left(1+9 r^{2}+4 r \cos \theta\right)}{1-2 r^{2}+81 r^{4}+8 r\left(1+9 r^{2}\right) \cos \theta+36 r^{2} \cos ^{2} \theta}<2-\alpha .
$$

If we let

$$
h(t)=\frac{\left(1-9 r^{2}\right)\left(1+9 r^{2}+4 r t\right)}{1-2 r^{2}+81 r^{4}+8 r\left(1+9 r^{2}\right) t+36 r^{2} t^{2}},
$$

then, we have

$$
h(t) \leq h(-1)=\frac{1-4 r+36 r^{3}-81 r^{4}}{1-8 r+34 r^{2}-72 r^{3}+81 r^{4}} \equiv \frac{g_{1}(r)}{g_{2}(r)} .
$$


Noting that $0<g_{1}(r)<1$, and $g_{2}(r)>g_{2}(1 / 13)=15876 / 28561$, we have

$$
h(t) \leq h(-1)<\frac{1}{g_{2}(r)}<\frac{28561}{15876}=2-\alpha,
$$

which implies that $\alpha=3191 / 15876=0.200 \ldots$

\section{REFERENCES}

[1] K. S. Padmanabhan, On the partial sums of certain analytic functions in the unit disc, Ann. Polon. Math. 23 (1970/1971), 83-92. MR 42\#488. Zbl 199.40501.

[2] G. Szegö, Zur theorie der schlichten abbilungen, Math. Ann. 100 (1928), 188-211.

Shigeyoshi OWa: Department of Mathematics, Kinki University, Higashi-OSaka, OSAKA 577-8502, JAPAN

E-mail address: owa@math .kindai .ac.jp 


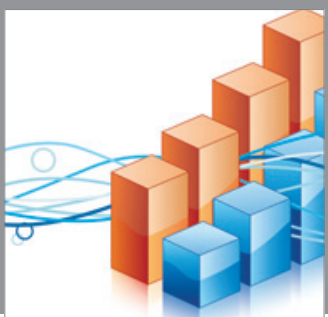

Advances in

Operations Research

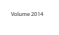

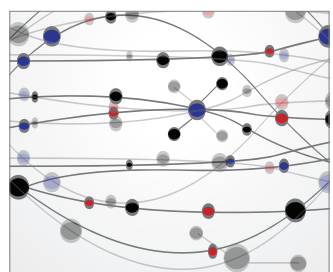

\section{The Scientific} World Journal
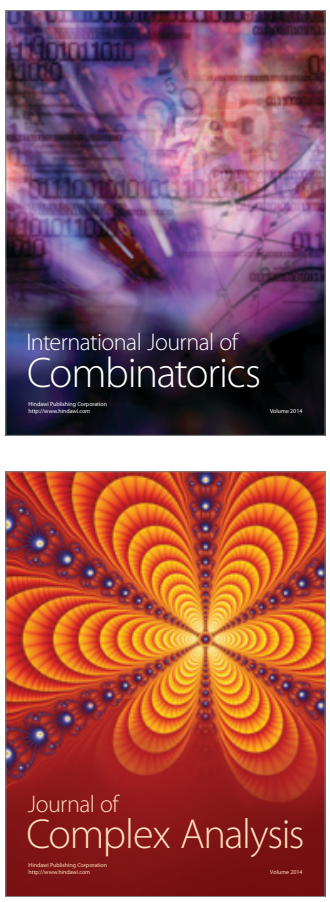

International Journal of

Mathematics and

Mathematical

Sciences
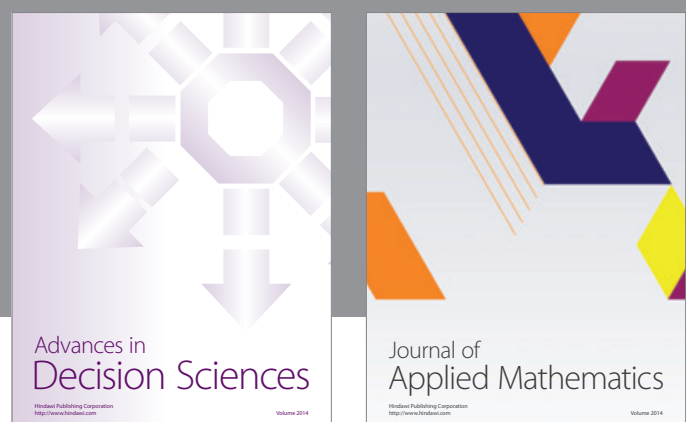

Journal of

Applied Mathematics
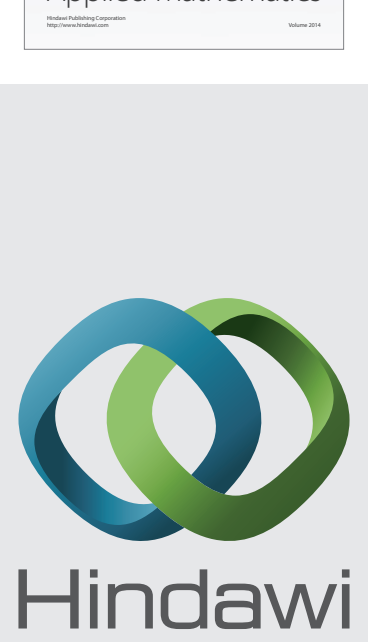

Submit your manuscripts at http://www.hindawi.com
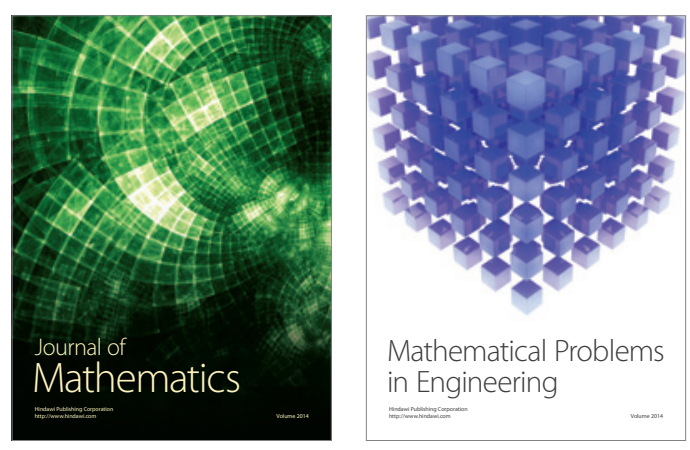

Mathematical Problems in Engineering
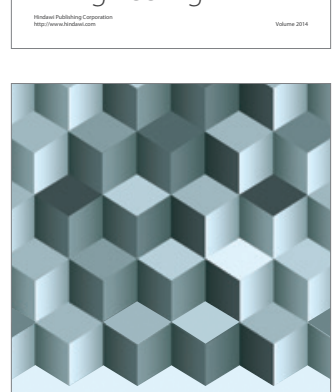

Journal of

Function Spaces
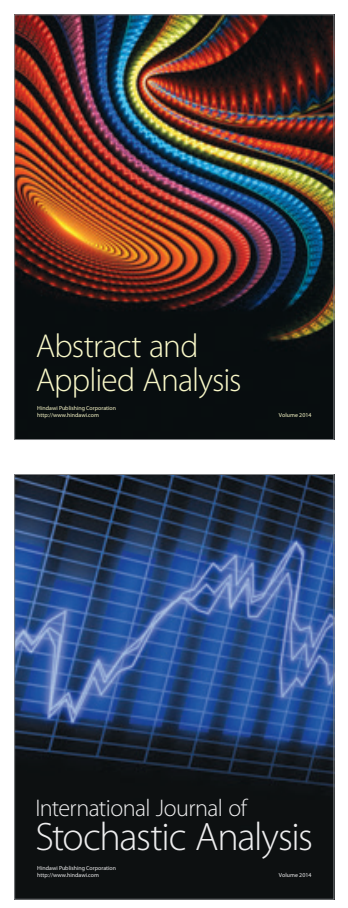

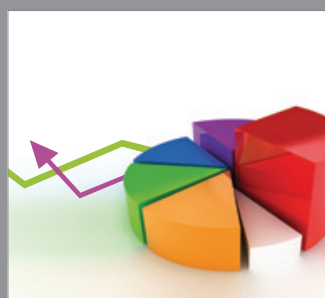

ournal of

Probability and Statistics

Promensencen
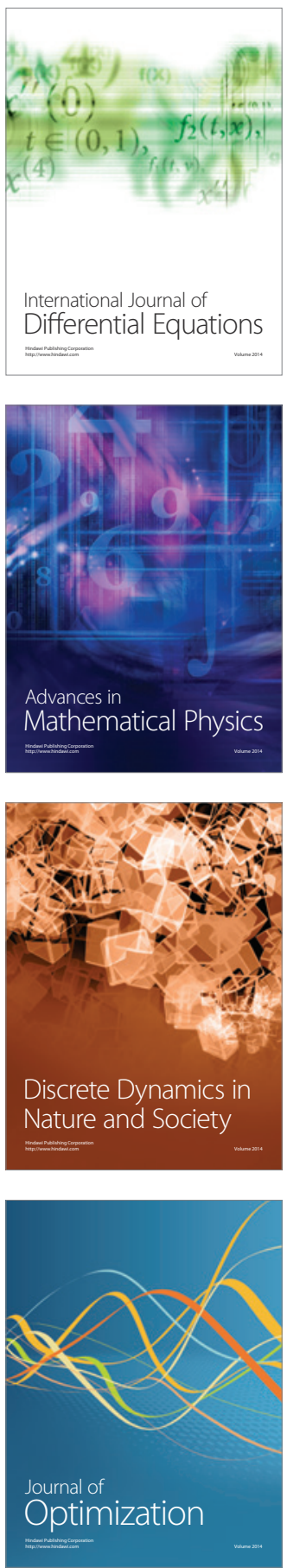